\title{
Potential Clinical Consequences of Antibodies to Animal-Derived Products in Surgery
}

\author{
Bruce Cronstein $^{*, a}$, Nigel Mackman ${ }^{\mathrm{b}}$ and Frederick A. Ofosu ${ }^{\mathrm{c}}$ \\ ${ }^{a}$ NYU School of Medicine, New York, NY, USA \\ ${ }^{b}$ University of North Carolina, Chapel Hill, NC, USA \\ ${ }^{c}$ McMaster University, and Senior Scientist, Canadian Blood Services, Hamilton, Ontario, Canada
}

\begin{abstract}
Many therapeutically effective animal-derived biopharmaceuticals and biomaterials, e.g., bovine thrombin, heparin and collagen, have been used successfully for decades during surgery, despite the potential formation of antibodies. Whether biological products are derived from human or animal sources or are recombinant proteins, an antigenic response can be provoked by many different factors inherent to biopharmaceuticals or biomaterials, including differences in amino acid sequences and glycosylation, impurities/contaminants in the biological preparations and patient-specific factors. Administration of products containing biological proteins can result in a range of reactions and the development of antibodies that can be serious but does not always have significant clinical consequences. Overall, only small proportions of treated patients develop severe reactions to these products. For example, out of the millions of patients treated with erythropoietin, 175 patients developed pure red cell aplasia, and of 4109 patients with injectable collagen implants, treatment-related adverse reactions (mostly localized swelling and erythema) occurred in $1.3 \%$ of patients. Despite their potential immunogenicity, physicians still use animal-derived products because of the beneficial effects on patient outcomes during surgery. Since retrospective clinical and immunogenicity assessments of these commonly used products is unlikely, the key for new animal-derived biopharmaceuticals is to carry out comprehensive immunogenicity assessments during clinical development before they reach the marketplace.
\end{abstract}

Keywords: Antibody formation, biological products, bovine, collagen, erythropoietin, fibrinogen, heart valve prosthesis, hemostasis, heparin, pericardium, porcine, thrombin, xenobiotics.

\section{INTRODUCTION}

Many effective animal-derived biopharmaceuticals and biomaterials have been used during surgical procedures for many years with great success, despite the potential for immunogenicity. Antibody formation can occur in response to all biopharmaceuticals, the frequency of which varies considerably [1].

Biopharmaceutical immunogenicity results as part of the body's normal immune reaction to a foreign protein [2] and is comparable to that which occurs when a vaccine is administered [1]. Antibodies are induced in 2 ways: by the classical immune response or by breaking immune tolerance [1].

Biopharmaceutical proteins can act as immunogens and stimulate the classical immune response [2]. Such proteins can incite a protective immune response, consisting of cellular and humoral components, that not only facilitates the elimination of the 'foreign antigen' but also commits to memory the specific foreign antigenic epitope to better prepare the immune system for future exposure to the same foreign antigen [3]. The initial or primary immune response to a protein antigen is its binding to specific receptors on circulating B cells. When an antigen binds to B cells, the cell is

*Address correspondence to this author at the NYU School of Medicine, NBV 16 16N1, 550 First Avenue, NY 10016, USA;

E-mail: Bruce.Cronstein@nyumc.org activated to proliferate and produce plasma cells that secrete antigen-specific antibodies (immunoglobulins, e.g., IgG and $\operatorname{IgM}$ ) that bind and inactivate the foreign protein [3] (Fig. 1a). B-cell activation also creates memory $B$ cells with the same antigen specificity that allows a rapid immune response to occur during future exposure to the same antigen. This secondary or enhanced recognition/memory immune response requires interaction with $\mathrm{CD} 4+$ helper $\mathrm{T}$ cells and occurs with prolonged or re-exposure to the same antigen (Fig. 1b). The secondary immune response may be important when patients are re-exposed to a substance that previously induced a primary immune response, such as re-exposure to a therapeutic biological protein [3].

Antibody formation in response to a biopharmaceutical protein or peptide can also be induced by breaking the immune tolerance to self-antigens [1]. For example, antibodies produced against a human homologue biopharmaceutical protein, e.g., a recombinant human protein such as interferon or erythropoietin (Epo), can expand recognition of or crossreact with the endogenous, homologous human proteins with similar epitopes and thus break the self-tolerance that normally exists. This leads to autoimmunity or the production of autoantibodies against the biopharmaceutical $[1,4,5]$. In the case of Epo, the net effect is the functional absence of Epo in patients with autoantibodies to Epo. In contrast to the neutralizing and non-neutralizing antibodies produced in the classic immune reaction to foreign proteins, these antibodies against human proteins often appear only after prolonged 
(a)

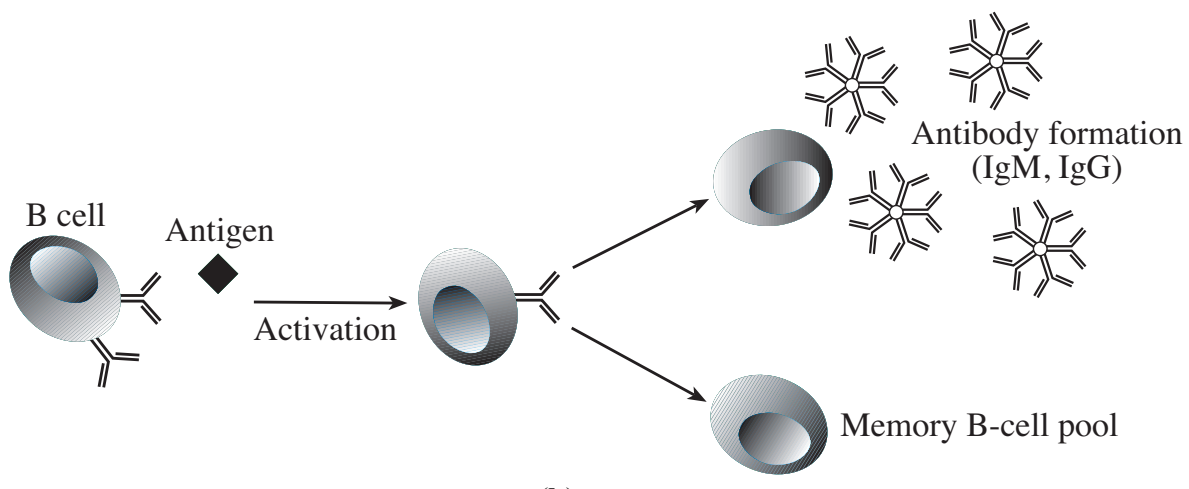

(b)

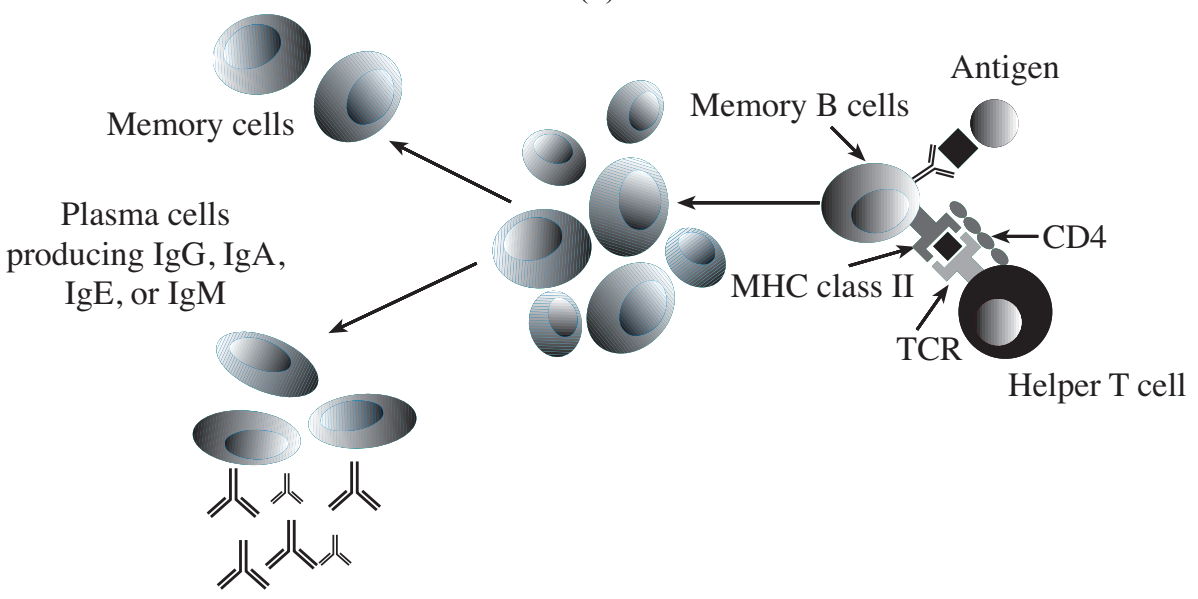

Fig. (1). Basic primary (a) and secondary (b) immune responses (modified after Porth, 1998; Koren 2002).

treatment and develop in fewer than $1 \%$ of patients. Typically, some of these autoantibodies disappear during treatment or when treatment with the offending biopharmaceutical protein ceases [1].

Antigenic responses can be provoked by many different factors inherent to biopharmaceuticals or biomaterials, whether derived from human or animal sources or recombinant proteins [6, 7] (Box 1). For example, the immune system's ability to recognize subtle differences between the amino acid and/or glycosylation sequences of endogenous human proteins and biopharmaceutical proteins is sufficient to provoke immune responses and the generation of antibodies. In the case of the recombinant biopharmaceutical Epo (epoetin) for example, which previously had an excellent safety record and had been prescribed to millions of patients without problems [6], the development of pure red cell aplasia (PRCA) associated with anti-Epo autoantibodies was found to have increased from a total of 3 cases between 1988 and 1998 to 175 for Eprex alone between 1998 and 2004 [4]. Patients developed PRCA within 9 months of exposure [4]. This led to the blocking of red blood cell maturation and differentiation in the affected patients [1]. The only explanation for this serious side effect appears to be subtle changes in the Eprex Epo molecule that occurred during manufacturing, formulation, handling, or distribution that were difficult to detect before hundreds of thousands of patients had been exposured to the product.
Box 1. Factors that Promote Antibody Production in Response to Administration of Biopharmaceutical Products $[1,6,7]$

\begin{tabular}{|c|c|}
\hline$\bullet$ & Variation in amino acid sequence \\
\hline$\bullet$ & Modification of amino acid side chains (e.g., oxidation or deamination) \\
\hline$\bullet$ & Glycosylation variation \\
\hline • & Differences in protein structure (e.g., proteolysis, partial denaturation) \\
\hline$\bullet$ & Contaminants and impurities \\
\hline$\bullet$ & Downstream processing and formulation \\
\hline & $\begin{array}{l}\text { Other immunogenic potential, such as route of application, dosing, } \\
\text { length of treatment, and patient's immune status }\end{array}$ \\
\hline
\end{tabular}

Variation in glycosylation is another possible factor that can contribute to immunogenicity associated with biopharmaceuticals as proteins produced in prokaryotes are not glycosylated. Two examples of non-glycosylated biopharmaceuticals are granulocyte-macrophage colony-stimulating factor and interferon $\alpha$. Non-glycosylation may induce production of neutralizing antibodies as these recombinant molecules are less soluble than the endogenous molecules or because the biopharmaceutical molecule presents epitopes that are normally hidden by glycosylation in the endogenous human protein $[8,9]$. In addition, immunogenic reactions to some biopharmaceuticals have been attributed to impurities 
or protein aggregates present in these preparations rather than structural changes in the therapeutic proteins. Generally speaking, the immunogenicity of the impurities present in the biopharmaceutical directly relates to its ability to provoke immunogenic responses.

The clinical consequences of antibody production to biopharmaceuticals are variable, however, and are not necessarily linked to the quantity of antibodies, but they may be closely correlated to the type of antibody produced. A1though some biotherapeutic proteins do not seem to be immunogenic, others induce the production of antibodies that can impact on clinical response, while others can induce antibodies that cross-react with endogenous proteins (Table 1).

Table 1. Immunogenicity of Some Recombinant Biopharmaceuticals

\begin{tabular}{|c|c|}
\hline Type of Response & Example of Biopharmaceutical \\
\hline Non-immunogenic & $\begin{array}{l}\text { - Granulocyte colony-stimulating factor } \\
\text { - Interferon (IFN)- } \gamma\end{array}$ \\
\hline Some clinical effect & $\begin{array}{l}\text { - Interleukin } 2 \\
\text { - Granulocyte-macrophage colony- } \\
\text { stimulating factor } \\
\text { - IFN- } \alpha \\
\text { - IFN- } \beta\end{array}$ \\
\hline Severe adverse events & $\begin{array}{l}\text { - Megakaryocyte growth and development } \\
\text { factor } \\
\text { - Erythropoietin }\end{array}$ \\
\hline
\end{tabular}

The main clinical consequence of antibody formation seems to be loss of product efficacy, which can sometimes be restored by increasing the product dose [1]. Antibodies produced against therapeutic products are of most concern when they neutralize endogenous proteins, which have essential biological roles, e.g., PRCA associated with Epo treatment [1]. Due to the variability of responses to biopharmaceuticals and the complexities of product comparisons, an extensive clinical history during administration of a biopharmaceutical becomes an important factor in the overall assessment of its safety profile during routine clinical practice.

This paper describes the immunogenicity of certain animal-derived biopharmaceuticals and biomaterials used in surgery, assesses the clinical consequences, and discusses the methods available for determining the potential immunogenicity of biopharmaceutical product during clinical development.

\section{ANIMAL-DERIVED BIOPHARMACEUTICALS USED IN SURGERY}

\section{Topical Hemostatic Agents and Tissue Sealants}

Excessive blood loss is a complication of surgery, physical trauma, and burns $[10,11]$, and surgeons can use many approaches to control bleeding depending on the procedure and site of bleeding. When mechanical or thermal hemostatic techniques fail or are impractical, surgeons often control bleeding using topical pharmacological interventions [12, 13], including animal-derived biopharmaceutical products that exert pharmacological effects at various points in the coagulation cascade $[1,6,14]$. These animal-derived proteins, such as bovine thrombin, have been used successfully for many years.

\section{Topical Thrombin}

Thrombin is the central protease of blood coagulation [15]. It converts fibrinogen to fibrin; activates blood platelets, factors VIII, V, XI, and XIII, and protein C; and has critical functions in the wound-healing process [16]. This is reflected in its wide use in surgery; it is as commonly used as saline $[16,17]$. Bovine thrombin is a biologically active topical hemostatic modifier that is used alone or in combination with other agents to promote and sustain hemostasis. It was first used for the treatment of surface bleeding in the 1930 s $[16,17]$. Thrombin is also used in both autologous and commercially available fibrin sealants [16].

As with any animal-derived protein, bovine thrombin may induce an immune response [18]. Many factors are thought to contribute to the immunogenicity of bovine thrombin in patients, from its xenogeneic origin to differences in the amino acid sequences or glycosylation between bovine and human thrombins [19]. Differences in glycosylation and amino acid sequences seem to be the most likely causes of the immunostimulatory properties of this therapeutic protein, because it is more heavily glycosylated than human thrombin and there is approximately a $10 \%$ difference between the amino acid sequences of human and bovine prothrombin [1].

Topical thrombin originates from bovine sources' therefore, some patients may develop antibodies against thrombin or any contaminating bovine coagulation factors, e.g., prothrombin, cardiolipin factor $\mathrm{V}$, and factor $\mathrm{Va}$, that may, on rare occasions, cross-react with endogenous human clotting factors $[14,19]$. Published reports have shown that the clinical impact of the development antibodies to topical thrombin preparations varies greatly, ranging from asymptomatic to laboratory abnormalities, such as prolonged prothrombin time (PT) and partial prothrombin time (PPT), to clinically relevant coagulopathies such as excessive postoperative bleeding in patients or thrombosis [19].

The development of anti-factor $\mathrm{V}$ and factor $\mathrm{Va}$ antibodies is likely to result from factor $\mathrm{V}$ and factor $\mathrm{Va}$ fragment contaminants in some topical bovine thrombin preparations [19]. As with many animal-derived protein products, improvements in the manufacturing process have reduced the levels of contaminants in newer bovine thrombin products. For example, with the inclusion of a membrane filtration step in the manufacturing process, the amount of factor Va light chain in the current bovine thrombin product is below the limit of detection using a semi-quantitative Western Blot assay [20].

The immunogenic response and adverse events in patients exposed to bovine thrombin during surgery has been studied in 2 trials with inconsistent findings. In a study involving 151 patients undergoing cardiac surgery, Ortel et al. showed that bovine thrombin was immunogenic and associated with an increased risk of adverse clinical outcomes [18]. Antibodies (both bound IgG and IgM) to thrombin and any contaminating coagulation proteins (including factor $\mathrm{V}$, factor $\mathrm{Va}$, and prothrombin), were detected using enzymelinked immunosorbent assay (ELISA). More than 95\% of 
patients developed a seropositive response to bovine coagulation proteins, and $51 \%$ had elevated antibody levels to corresponding human coagulation proteins after exposure to bovine thrombin. Patients with elevated antibodies to human coagulation factors were determined to be more likely to suffer coagulation abnormalities. Contrasting results were found in another study conducted by Winterbottom et al. [21]. In this study, 309 patients who were exposed to bovine thrombin during cardiac, vascular, or spinal surgery were tested for bovine thrombin and factor $\mathrm{Va}$ antibodies using ELISA. In this study, a similar number of patients $(19 \%)$ were found to be seropositive for bovine thrombin but only $30 \%$ were seropositive for bovine factor Va. Unlike Ortel et $a l$, this study reported that the presence of bovine thrombin or factor Va antibodies preoepratively or postoperatively was not associated with increased clinical adverse outcomes, including coagulopathy [21]. While the association between topical bovine thrombin and coagulopathy does exist, a definitive and consistent association has not been made.

In addition to bovine thrombin and coagulation protein antibodies, other antibodies associated with the clinical use of bovine thrombin have been reported, including antibodies to galactose- $\alpha 1-3$ galactose (gal $\alpha 1-3 \mathrm{Gal}$ ) and antiphospholipid antibodies [22, 23]. Many glycoproteins in topical thrombin preparations have been found to contain the gal $\alpha 1-$ $3 \mathrm{Gal}$ epitope [22]. Gal $\alpha 1-3 \mathrm{Gal}$ is expressed in large amounts in all mammals (including rabbits, pigs, and cows) except humans and old-world apes [22]. Interestingly, natural antigala1-3Gal antibodies are often found in people who have never been exposed to biopharmaceuticals, including topical bovine thrombin. Gal $\alpha 1-3 \mathrm{Gal}$ is recognized by natural antibodies in normal people that mediate complement activation and destruction of xenogeneic tissues [22]. Schoenecker et al. determined anti-gala1-3Gal IgG and IgM antibody levels (using ELISA) in 63 patients following coronary artery bypass grafting (CABG) [22]. Following exposure to topical bovine thrombin, anti-gal $\alpha 1-3 \mathrm{Gal} \mathrm{IgG}$ antibodies increased to levels 10 times greater than those of natural anti-gal $\alpha 1-$ $3 \mathrm{Gal}$ antibodies in individuals who have never been exposed to bovine thrombin or any other animal-derived biopharmaceutical [22]. It is apparent; therefore, that gal $\alpha 1-3 \mathrm{Gal}$ may account in part for the immunogenicity seen with bovine thrombin [22].

It is difficult to determine what percent of antibodies developed against a biopharmaceutical are pathogenic; however, clinical experience demonstrates that $20 \%$ to $90 \%$ of patients treated with topical bovine thrombin subsequently have detectable levels of antibodies, although these patients rarely develop coagulopathies [18, 21]. Precise assessment of the use of bovine thrombin in surgical cases is unavailable, although it is conservatively estimated that at least 500,000 Americans are exposed to some type of topical bovine thrombin preparation each year [22]. The incidence of clinically significant antibody-mediated adverse events to topical bovine thrombin has been low since its use became widespread in the United States after receiving US Food and Drug Administration (FDA) approval in the 1970s [19].

\section{Gelatin-Based Products}

Other animal-derived absorbable topical hemostatic agents are often used as adjunctive therapy during surgery
[14]. Products containing sterile porcine or bovine gelatin are available in a variety of formulations and are indicated to provide hemostasis at bleeding surfaces [24, 25]. These biologically passive products are believed to initiate clotting through contact activation and by providing a scaffold for platelet aggregation, although the exact mechanism is unknown [14]. Gelatin-based products may be used alone or in combination with thrombin. In combination, the 2 components work synergistically to produce a stable clot at the bleeding site that limits blood loss. These components provide a matrix around which a clot can form and localize active thrombin to promote rapid hemostasis [14]. In the case of spinal surgery, for example, it is essential to achieve hemostasis during surgical procedures within the spinal canal, where bleeding of only a few milliliters of blood may cause devastating neurologic damage. The use of chemical hemostasis is preferred to bipolar cautery because of its superior efficacy in controlling diffuse capillary bleeding [12]. Despite the complications of absorbable porcine gelatin and regenerated oxidized cellulose as hemostats in intraspinal surgery, Sabel and Stummer concluded that these agents are considered safe and beneficial and the proper administration should prevent serious adverse patient outcomes [12].

A search of the literature revealed no reports of immunogenic reactions to these gelatin-based products, although Nakamura et $\mathrm{al}$. reported that poorly hydrolyzed bovine gelatin was immunogenic when administered with alum adjuvant in vaccines [26]. Most anaphylactic and urticarial reactions to gelatin-containing measles, mumps, and rubella (MMR) monovalent vaccines were associated with IgE-mediated gelatin allergy [27]. A retrospective study has shown that among 26 cases of anaphylaxis, $6(27 \%)$ were positive for anti-gelatin IgE antibodies [26]. The reported incidence of anaphylactic reactions, however, is 1.8 per million doses of MMR vaccine and this has not increased since the introduction of the gelatin-containing diphtheria-tetanus-acellular pertussis (DTaP) vaccine in 1998 [26]. DTaP immunization histories suggest that the gelatin in $\mathrm{DTaP}$ vaccines may have a causal relation with the development of this allergy [27].

\section{Fibrin Glues and Sealants}

Fibrin glues and sealants are often applied topically to promote hemostasis when electrocautery, sutures or metal clips, and staples are not appropriate for delicate surgery on small yet functionally vital tissues and structures [28]. The 2 main components of these products-purified human fibrinogen and bovine or human thrombin-interact to form a fibrin network when applied [28]. Bovine aprotinin, a plasmin inhibitor, may be added to prolong the in vivo life of these products [29]. Although the principal application of fibrin sealants is to achieve hemostasis, they can also be used to seal air, bile, and lymphatic leaks and as glue and matrix for local drug delivery [30].

Various adverse effects have been reported with fibrin glues and agents, particularly during specific surgical procedures (laminectomy, craniotomy, lobectomy, and nasal surgery), with some rare adverse effects, such as paralysis, being quite severe [14]. Adverse events relevant to this discussion, however, have involved products that contain bovine thrombin and include allergic reactions and the development of inhibitory antibodies to bovine thrombin and coagulation 
proteins that interfere with the coagulation process [14] as well as Gal $\alpha 1-3 \mathrm{Gal}$ and cardiolipin, as described above.

Bovine aprotinin is often used as the fibrinolysis inhibitor in these products and is highly immunogenic in humans [30]. Indeed, anti-aprotinin IgG antibodies have been shown to be present in $80 \%$ of patients after exposure to local aprotinin during cardiac surgery [31]. In addition, after exposure to fibrin sealants during pediatric cardiac surgery, $39 \%$ of patients had anti-aprotinin IgG antibodies and $8 \%$ had IgE antibodies [32]. An immune response to bovine aprotinin can result in IgE-mediated type I or IgG-mediated type III hypersensitivity [30]. The incidence of anaphylaxis to fibrin sealant is estimated to be 0.5 per 100,000 applications; however, assuming that $90 \%$ of cases go unreported, the true figure is likely to be closer to 5 per 100,000 applications [30].

When considering the use of bovine aprotinin-containing products, it is important to assess the risk of immunogenicity. Although hypersensitivity reactions to bovine aprotinin are rare, the clinical consequences for some patients can be very serious [30]. Adverse effects associated with bovine aprotinin immunogenicity range from mild skin rashes to fatal anaphylaxis after local application. Although there are tests available to assess the likelihood of an immune response to aprotinin, only tests for specific anti-aprotinin $\operatorname{IgE}$ and IgG antibodies (ELISAs) are available in clinical practice, but none are used routinely [30].

\section{Collagen}

Collagen is a tissue-derived material used in a variety of biomaterials, including topical hemostatic agents [13]. Collagen-based absorbable hemostatic agents provide hemostasis through contact activation and the promotion of platelet aggregation as a direct effect of contact between blood and the agent's collagen [14]. After platelet aggregation begins, platelet degranulation occurs as does platelet-dependent coagulation [14].

Collagen consists of 3 polypeptide chains, called tropocollagen, arranged in a helical structure and a non-helical portion composed of telopeptides [13]. The antigenic determinants of collagen can be classified into 3 categorieshelical, central, and terminal [33]. The antigenicity of collagen is linked intimately with the concepts of self-tolerance and interspecies variation. The terminal telopeptides are the component of collagen with the highest interspecies variability, and some evidence suggests that the immunogenic potential of collagen is strongly reduced by proteolytic digestion of these telopeptides [13]. Other evidence, however, suggests that removal of telopeptides provides no immunologic benefit of clinical significance [33]. Immune responses to collagen involve both antibody formation and an enhanced recognition component, but their relative contributions are not yet known [33]. Between $2 \%$ and $4 \%$ of the total population have an inherent immunity (allergy) to bovine collagen, but this is a relatively low incidence compared with other common allergies [33]. Skin tests are available that can identify such people before treatment with collagenbased products is initiated, although a further $1 \%$ to $2 \%$ of people will develop immune reactions after exposure to a collagen hemostatic product [33]. Overall, collagen has low antigenicity and immunogenicity [33], but immunogenicity does vary between the different hemostatic and injectable collagens [13]. As with all bovine- and porcine-derived materials, development of anti-Gala1-3Gal antibodies can often also occur, as described above.

\section{Bovine or Porcine Heparins}

Heparin is used prophylactically to prevent postoperative deep vein thrombosis (DVT) in patients undergoing orthopedic, general, and gynecological surgery $[34,35]$. It is also the anticoagulant most commonly used to prevent clotting during cardiac or vascular surgery [36]. Heparin may be extracted from porcine intestinal mucosa or bovine lung, where it is bound to histamine and stored in the mast cell granules $[35,36]$. The purified, unfractionated product is a heterogeneous mixture of glycosaminoglycans with an average molecular weight of 12000 [36].

Heparin-induced thrombocytopenia (HIT) is a potentially serious immune-mediated adverse response to heparin and is associated with an increased risk of thrombosis that can result in loss of limbs or death $[36,37]$. Up to $8 \%$ of patients treated with heparin will develop the anti-platelet factor 4 (PF4) antibodies associated with HIT, and $1 \%$ to $5 \%$ of patients will progress to develop HIT with thrombocytopenia [37]. Despite thrombocytopenia, bleeding is rare, but HIT is strongly associated with thrombosis, with a mortality of about $20 \%$ to $30 \%$ and permanent disability by amputation, stroke, or other causes in a further $20 \%$ to $30 \%$ [37].

The antigenic mechanism of HIT has been extensively reviewed by Franchini [37]. Briefly, the principal antigen is the complex of the highly acidic heparin and highly basic platelet factor 4 (PF4). PF4 is normally stored in platelets and is released into the circulation when platelets are activated [37]. The heparin-PF4 complex presents neoepitopes that act as immunogens and provoke production of antibodies [37]. This results in the production of IgG antibodies directed against the heparin-PF4 complex. The IgG antibodies bind to the complex on the platelet surface through the Fab region [37]. The Fc portion of the HIT antibody binds to the platelet $\mathrm{Fc}$ receptor, and this triggers activation and aggregation of platelets, which release more PF4 to perpetuate the cycle of heparin-induced platelet activation [37]. This antibody-mediated activation of platelets also leads to the production of prothrombotic platelet microparticles that promote coagulation. The presence of heparin-like molecules (heparan sulfate) on the surface of endothelial cells means that HIT-antibody-PF4-heparan sulfate complexes on the endothelial surface may induce expression of the procoagulant molecule tissue factor with further coagulation activation [37]. Thrombocytopenia results from clearance of activated antibody-coated platelets [37]. The overall result is intravascular platelet activation, thrombocytopenia, and potential thromboembolic complications [38].

Francis et al. found that bovine heparin might be associated with a higher rate of heparin-PF4 antibody formation than porcine heparin [39]. In 207 patients undergoing firsttime coronary artery bypass graft (CABG) surgery with or without cardiopulmonary bypass, the overall incidence of anti-heparin-PF4 antibody formation was 42\% [39]. Six $(2.9 \%)$ patients were positive postoperatively and one of these developed clinical HIT [39]. When these 6 patients were excluded, seroconversion rates were $44.4 \%(44 / 99)$ with bovine heparin and $30.6 \%(33 / 108)$ with porcine hepa- 
rin $(P=.041)$ [39]. Most patients who produced antibodies ( $90 \%$ bovine, $85 \%$ porcine) seroconverted after postoperative day 2 [39]. No differences in postoperative platelet counts occurred, and only one patient developed thrombosis associated with seroconversion without also developing thrombocytopenia [39]. The authors concluded that there is a high frequency of anti-heparin-PF4 antibodies after CABG and that the incidence is significantly higher with bovine than porcine heparin [39].

In contrast, data from an earlier study by Konkle et al. did not support these findings [40]. In 98 patients, no significant difference in patient positivity for antibodies was seen postoperatively with the serotonin release assay $(12 \%$ in both groups) or the heparin-PF4 ELISA ( $29 \%$ with porcine heparin, $35 \%$ with bovine heparin) postoperatively [40]. There was also no significant difference between preoperative or postoperative platelet counts or thromboembolic complications [40]. The study did confirm that an unexpected number of patients had developed antibodies before surgery was performed and that levels of these antibodies increased in most patients during the perioperative period [40]. Although some patients developed thrombotic complications, there was no correlation with antibody formation or platelet count at the time of the event and thus no diagnosis of HIT or HIT with thrombosis was made [40].

The clinical criteria for diagnosing HIT are thrombocytopenia (platelet count $<100 \times 10^{9}$ or a $50 \%$ drop from patient's baseline level), exclusion of other causes of thrombocytopenia, and disappearance of thrombocytopenia when heparin treatment is stopped [37]. The presence of antiheparin/HIT antibodies can be determined by functional tests and immunoassays. Functional tests, such as heparin-induced platelet aggregation (HIPA) and the serotonin release assay (SRA), measure platelet activity. Although the SRA is considered to be the gold standard, it is more difficult to perform and is not widely available, so the HIPA test is more commonly used [37]. ELISAs can detect the PF4/heparin complex antibody. These immunoassays are relatively easier to perform and more sensitive than functional tests; however, functional tests may be more effective at detecting clinically significant HIT [37]. These tests, however, tend not to be routinely available in clinical practice. Although HIT does not invariably recur during subsequent re-exposures to heparin, future use of heparin is contraindicated in these patients [37]. Again, as with all bovine- and porcine-derived materials, development of anti-Gala1-3Gal antibodies can often also occur, as described above.

\section{Low-Molecular-Weight Heparin}

Low-molecular-weight heparin (LMWH) was introduced in the late 1980s for the prevention and treatment of DVT [41]. LMWH is used to prevent DVT in a variety of clinical situations, including general and hip or knee replacement surgeries, and is as effective as unfractionated heparin (UFH) in preventing DVT [41]. LMWH can also be used as an adjunct therapy while waiting for oral anticoagulation to take effect during the treatment of DVT [41].

Early reports suggested that short-term use of LMWH was not associated with HIT [41] and, by 2004, only a few cases of HIT had been reported following treatment with LMWH [38]. In a study of 180 patients treated for suspected
HIT, the condition was confirmed in 59 patients; 57 had high levels of antibodies to heparin-PF4 complexes and 2 had antibodies to interleukin 8 [38]. These patients had been treated with LMWH $(\mathrm{n}=11)$ unfractionated heparin (UFH) alone $(n=34)$, or UFH combined with LMWH $(n=14)$. In patients who received LMWH, the interval to onset of HIT was longer, and severe thrombocytopenia (platelet count $\left.<15 \times 10^{9} / \mathrm{L}\right)$ was more frequent $(P=.04)$ than in those who received UFH. Thrombosis was equally observed in both patient groups [38]. In another study, the frequency of HIT was lower in patients who received prophylaxis with LMWH for 9 months after hip or knee replacement than in those who received UFH ( $0 \%$ vs $5.2 \%, P<.001)$. Overall, total thrombosis was significantly more frequent in the LMWH patient population than the UFH group [42].

A review by Walenga et al. suggested reasons for the reported differences in the frequencies of HIT associated with LMWH and UFH [43]. Similar mechanisms were suggested for the pathophysiology of HIT caused by UFH and LMWH because their chemical structures are similar. However, the smaller size of LMWH means that it cannot interact with both PF4 and platelets as efficiently as the larger UFH [43]. This translates to a 2- to 3-fold reduced risk of immune sensitisation (antibody generation and occurrence of clinical HIT) [43]. In addition, LMWH-induced HIT antibodies are more often IgA and IgM, while IgG is more commonly generated in HIT induced by UFH. The differences in the types of antibodies generated in response to LMWH and UFH may explain the greater tendency for clinical HIT after UFH [43].

\section{Animal-Derived Biomaterials}

A biomaterial is any natural or manmade material that augments or replaces a natural function within a living organism. The Romans, Chinese, and Aztecs used the very first biomaterials more than 2000 years ago in the form of gold used in dentistry [44]. With the development of synthetic polymer systems over the past few decades, biomaterials have begun to find broad applications in modern medicine [45]. Indeed, biomaterials have played an enormous role in the success of medical devices and drug delivery systems [45] and are included in a vast array of products, such as synthetic replacements for biological tissues, materials for specific medical applications, and materials for diagnostics [45].

\section{Collagen}

As described above, collagen is used as a component of topical hemostats. Bovine collagen is also used in a number of medical biomaterials, including sutures, pericardial substitutes, wound and burn dressings, vascular graft coating, and soft-tissue augmentation, as well as the treatment of urinary incontinence [13]. Collagen has high tensile strength, high affinity with water, low antigenicity, high absorbability, and good cell compatibility. Furthermore, collagen enhances tissue regeneration, influences cell differentiation, and activates platelets. These properties are controllable by physical and chemical modifications and availability of variously shaped fabrications [13]. It is one of the best materials for use as a scaffold in tissue engineering and, with modification, can be used in a variety of fields [13]. Injections of bovine collagen are used in plastic surgery to improve the 
skin's contour and fill out depressions in the skin caused by scarring, injury, or aging [46].

Immunogenicity to implanted collagen has been shown to occur, but current published literature is sparse. One study has shown that 5 of $68(7 \%)$ patients implanted with a bovine collagen-coated vascular graft developed anti-bovine collagen antibodies [13]. A skin test in one patient who had a systemic allergic reaction to a collagen implant during laparoscopic cholecystectomy showed a notable allergic reaction, while an IgE radioallergosorbent test against bovine serum albumin was within the normal limits [36]. Immunologic responses to composites of pepsin-solubilized bovine type I collagen and calcium phosphates have been limited to elevated levels of circulating antibodies to collagen, with no reported effect on the efficacy of the implant itself [33].

Although data on immunologic responses to bovine and porcine dermal collagens used as dermal substitutes for wound cover and wound closure are meager, no collageninduced adverse immunologic responses to non-allograft dermal substitutes have been documented despite the numerous collagen sources and varied extraction methods used [33]. Fibrillar collagen-containing products used for hemostasis have marginally higher incidences of induced allergy to collagen, and a granulomatous foreign body reaction was observed after application of a microfibrillar collagen hemostat in the spleen, but such devices often contain noncollagenous protein contaminants (including the glycoprotein Gala1-3Gal), which may be the main immunogenic components of collagen-based hemostats [33].

Screening for collagen allergy/immunogenicity using a readily available and simple skin test should be carried out routinely before collagen is implanted in patients [33].

\section{Biologic Valves and Bovine Pericardial Tissue}

Biologic (or bioprosthetic) valves made of bovine or porcine tissue may be used in patients requiring heart valve replacement surgery [47]. Porcine valves consist of natural porcine aortic valves sewn to a metal wire stent and covered with cloth made of polyethylene terephthalate fibers [47, 48]. Bovine valves are made of bovine pericardial tissue mounted on a frame of molded silicone rubber [47]. Biologic valves are more similar to the natural valve than mechanical valves and may not require lifelong anticoagulant therapy (unless the patient has other conditions that warrant such treatment).

Bovine pericardial tissue has other uses, e.g., in the surgical treatment of emphysema $[49,50]$ and in extra-cardiac lateral tunnel Fontan procedures to create a conduit from the inferior vena cava to the artery in children with complex congenital heart defects [51]. It is also used as a penile prosthesis lining [52], a wrapping material for hydroxyapatite implants in patients undergoing enucleation for uveal melanoma [53] and in the repair of incisional hernias [54].

The most common cause of bioprosthesis failure is stiffening of tissue due to the buildup of calcium, which can restrict blood flow through the valve or cause tears in the valve leaflets. The use of biologic heart valves decreased in the 1990s because of the high incidence of failure due to tissue degeneration or tearing [55]. As younger patients have greater metabolism of calcium, bioprostheses tend to last longer in older patients [48], and premature degeneration of biologic valves has limited their use in younger patients in recent years [56]. Inflammatory and immune reactions were thought to mediate both calcification and biodegradation [57], but the exact immune response has remained unclear [56].

\section{METHODS OF ASSESSING IMMUNOGENICITY}

Assessing antibody responses should be an integral part of the clinical development of biopharmaceutical products and form part of the safety evaluation. During clinical development, thorough and appropriate detection, quantification, and characterization of antibody responses is needed to assess potential immunogenicity. This requires the implementation of anti-product antibody immunoassays during preclinical and clinical trials [58]. Indeed, this is now a requirement of most regulatory authorities, including the European Medicines Agency and the US FDA [59].

Numerous assays are available that will detect the presence of antibodies to biopharmaceutical products $[7,58]$. Methods for detecting antibodies include immunochemical tests e.g. binding assays (solid phase binding immunoassays such as ELISA) and immunoblotting, and biophysical tests, e.g., surface plasmon resonance (SPRs) and functional bioassays. Each assay has its own advantages and disadvantages (Table 2). A comprehensive assessment of a product's immunogenicity requires a strategy that utilizes a combination of these methods [7]. Extrapolations of published data comparing one particular biological therapeutic to another often can be misleading and invalid due to the inadequacy of the antibody evaluation methods used to assess immunogenicity. Similarly, conclusions about the immunogenicity of products drawn by comparing data from multiple studies with different products can also be misleading. Comparative immunogenicity of similar biological products should be determined only from studies appropriately designed for that purpose $[7$, 58].

In studies determining the immunogenicity of single or multiple biopharmaceutical products, the most common methodology is to quantitatively assess the antibodies produced after the products are administered to patients. Different antibody capture probes with variable specificities are currently used. It is critical for comparability that probes with similar and very specific capture abilities be used across multiple antibody assessment methods. These methods and probes should be used in a crossover manner with different products and appropriate controls in order to assess the specificity of the antibody capture probe and any crossreactivity between proteins $[7,58]$. Overall, assays for detecting production of antibodies directed against biopharmaceutical products in patients require assay standardisation and validation in order to obtain results that facilitate meaningful comparisons of the antigenicity of similar products.

Few tests, however, are available commercially to confirm possible immunogenicity in patients during routine clinical practice. Most antibody tests are for use in the research setting only. Immunogenicity to biopharmaceuticals, such as bovine thrombin, fibrin sealants, etc, that arises after surgery tends to be diagnosed clinically; ie, evidence of an allergic reaction or coagulopathies in the case of thrombin. Confirmation of the presence of antibodies to the biopharmaceutical is rarely carried out. Even if the presence of antibodies is determined in 
Table 2. Advantages and Disadvantages of Tests Used to Identify Antibodies (Adapted from Wadhwa, et al. 2003) [7]

\begin{tabular}{|c|c|c|c|}
\hline Test & How it Works & Advantages & Disadvantages \\
\hline $\begin{array}{l}\text { Binding assay, } \\
\text { e.g., ELISA }\end{array}$ & $\begin{array}{l}\text { Uses an antigen prepa- } \\
\text { ration to detect which } \\
\text { antibodies will bind }\end{array}$ & $\begin{array}{l}\text { - } \text { Quick to perform } \\
\text { - Relatively easy to use } \\
\text { - High throughput } \\
\text { - Good sensitivity }\end{array}$ & $\begin{array}{l}\text { - Spurious binding can occur } \\
\text { - 'Low-affinity' antibodies often undetected } \\
\text { - Antigen immobilization may alter protein conformation }\end{array}$ \\
\hline Immuno-blotting & $\begin{array}{l}\text { Determines an anti- } \\
\text { body's specificity for } \\
\text { protein in an antigen } \\
\text { preparation }\end{array}$ & $\begin{array}{l}\text { - Establishes specificity/reactivity of an } \\
\text { antibody } \\
\text { - Good sensitivity }\end{array}$ & $\begin{array}{l}\text { - Non-quantitative } \\
\text { - Low throughput } \\
\text { - SDS-PAGE may effect antibody's antigen binding }\end{array}$ \\
\hline SPR & $\begin{array}{l}\text { Establishes real-time } \\
\text { antigen-antibody inter- } \\
\text { actions }\end{array}$ & $\begin{array}{l}\text { - Automated } \\
\text { - Establishes antibody binding affinity, } \\
\text { isotype, specificity and concentration } \\
\text { - Detects 'low-affinity' antibodies }\end{array}$ & $\begin{array}{l}\text { - Antigen immobilization may alter protein conformation } \\
\text { - Less sensitive than binding assays } \\
\text { - Expensive }\end{array}$ \\
\hline Bioassay & $\begin{array}{l}\text { Determines an anti- } \\
\text { body's ability to neu- } \\
\text { tralize an antigen }\end{array}$ & $\begin{array}{l}\text { - Identifies neutralizing potential } \\
\text { - Possible correlation with clinical re- } \\
\text { sponse }\end{array}$ & $\begin{array}{l}\text { - Variable results } \\
\text { - Difficult to validate } \\
\text { - Time-consuming }\end{array}$ \\
\hline
\end{tabular}

the clinic, additional tests will rarely be used to ascertain the type of antibody and/or how it affects the patient clinically. Currently, some specific antibody immunoassays, such as anti-aprotinin $\operatorname{IgE}$ and $\operatorname{IgG}$ antibody ELISAs, are available in clinical practice, but none are used routinely. Simple-to-use collagen allergy skin tests are available, and all patients should undergo such testing prior to receiving collagen-based products [33].

Inevitably, immunogenicity can be expected with all animal-derived biopharmaceuticals [7]. The key objective is to gain a thorough understanding of the antibodies that can be induced against any therapeutic product and to be aware of the clinical consequences the antibodies may cause. Feasibly, a comprehensive strategy of immunogenicity testing will occur during early clinical development and not on a patient-bypatient basis after the product has reached the market. A full immunogenicity profile on the product can provide surgeons and patients with a better understanding of the risks, if any, that may be involved.

\section{CONCLUSION}

The medical use of animal proteins has a long history that started with the development of vaccines and immune sera from animals more than a century ago and was followed by the introduction of porcine and bovine insulins some decades later [1]. The foreign nature of the proteins leads to the occurrence of immunogenic reactions, including occasionally severe anaphylaxis. It was anticipated that the introduction of humanderived and later recombinant human homologues would avoid immunogenicity. Human biological medicines, however, whether derived from human plasma or from recombinant DNA technology, nearly always induce immunogenicity as well [1]. Indeed, immunogenic reactions to biopharmaceuticals are common, and many protein therapeutics currently in clinical use elicit some level of immune response, but the individual responses vary in their clinical implications. The production of antibodies is not necessarily associated with significant clinical sequelae and, for the most part, antibody production does not present serious problems [2]. In most cases, the type of antibodies produced, not the quantity of antibody developed, determines the clinical consequences.
Despite the immunogenic potential, physicians continue to use animal-derived products because the beneficial effects outweigh the possible risk in terms of patient outcomes. Overall, only a limited number of patients develop severe reactions that warrant avoidance of the product. In the future, computerdriven modeling followed by in vitro and/or in vivo testing of potentially immunogenic epitopes may predict and help avoid, or at least minimize, immune responses to foreign therapeutic products [2]. In the meantime, however, products that contain animal-derived proteins are generally more beneficial than harmful in the vast majority of patients.

\section{REFERENCES}

[1] Schellekens H. Immunogenicity of therapeutic proteins. Nephrol Dial Transplant 2003; 8(7): 1257-9.

[2] Koren E, Zuckerman LA, Mire-Sluis AR. Immune response to therapeutic proteins in humans - clinical significance, assessment, and prediction. Curr Pharma Biotechnol 2002; 3(4): 349-60.

[3] Sommers C. Immunity and inflammation. In: Porth CM, Ed. Pathophysiology: concepts of altered health states. Philadelphia, PA, Lippincott-Raven; 1998.

[4] Bennett CL, Luminari S, Nissenson AR, et al. Pure red-cell aplasia and epoetin therapy. N Engl J Med 2004; 351(14): 1403-8.

[5] Casadevall N, Nataf J, Viron B, et al. Pure red-cell aplasia and antierythropoietin antibodies in patients treated with recombinant erythropoietin. N Engl J Med 2002; 346(7): 469-75.

[6] Schellekens H. Bioequivalence and the immunogenicity of biopharmaceuticals. Nat Rev Drug Discov 2002; 1(6): 457-62

[7] Wadhwa M, Bird C, Diliger P, et al. Strategies for detection, measurement and characterization of unwanted antibodies induced by therapeutic biologicals. J Immuno Meth 2003; 278(1-2): 1-17.

[8] Gribben JG, Devereux S, Thomas NS, et al. Development of antibodies to unprotected glycosylation sites on recombinant human GMCSF. Lancet 1990; 335(8687): 434-7.

[9] Karpusas M, Whitty A, Runkel L, Hochman P. The structure of human interferon-beta: implications for activity. Cell Mol Life Sci 1998; 54(11): 1203-16.

[10] Block JE. Severe blood loss during spinal reconstructive procedures: the potential usefulness of topical hemostatic agents. Med Hypotheses 2005; 65(3): 617-21.

[11] Renkens KL Jr, Payner TD, Leipzig TJ, et al. A multicenter, prospective, randomized trial evaluating a new hemostatic agent for spinal surgery. Spine 2001; 26(15): 1645-50.

[12] Sabel M, Stummer W. The use of local agents: Surgicel and Surgifoam. Eur Spine J 2004; 13(suppl1): S97-101.

[13] Tomizawa Y. Clinical benefits and risk analysis of topical hemostats: a review. J Artif Organs 2005; 8(3): 137-42.

[14] Gabay M. Absorbable hemostatic agents. Am J Health-Syst Pharm 2006; 63(13): 1244-53. 
[15] Davie EW, Kulman JD. An overview of the structure and function of thrombin. Semin Thromb Hemost 2006; 32(suppl 1): 3-15.

[16] Lundblad RL, Bradshaw RA, Gabriel D, Ortel TL, Lawson J, Mann KG. A review of the therapeutic uses of thrombin. Thromb Haemost 2004; 91(5): 851-60.

[17] Price BD. General patient care and safety. In: Caruthers BL, Price P, Junge TL, Price BD, Eds. Surgical technology for the surgical technologist: a positive care approach. Albany, NY, Delmer Thompson Learning. 2001: 118-49.

[18] Ortel TL, Mercer MC, Thames EH, Moore KD, Laswon JH. Immunologic impact and clinical outcomes after surgical exposure to bovine thrombin. Ann Surg 2001; 233(1): 88-96

[19] Lawson JH. The clinical use and immunologic impact of thrombin in surgery. Semin Thromb Hemost 2006; 32(suppl 1): 98-110.

[20] Terrab A, Pawlak D, Spaay P, Hoppensteadt D, Fareed J. Further removal of factor $\mathrm{V}$ related antigen from bovine thrombin by utilizing a membrane-filtration step. Clin Appl Thromb Hemost 2008; 14(2): $135-40$.

[21] Winterbottom N, Kuo JM, Nguyen K, et al. Antigenic response to bovine thrombin exposure during surgery: a prospective study of 309 patients. J Appl Res Clin Exp Ther 2002; 2: 1-12.

[22] Schoenecker JG, Hauck RK, Mercer MC, Parker W, Lawson JH. Exposure to topical bovine thrombin during surgery elicits a response against the xenogeneic carbohydrate galactose alpha1-3galactose. J Clin Immunol 2000; 20(6): 434-44.

[23] Su Z, Izumi T, Thames EH, Lawson JH, Ortel TL. Antiphospholipid antibodies after surgical exposure to topical bovine thrombin. J Lab Clin Med 2002; 139(6): 349-56.

[24] Johnson \& Johnson Wound Management. Essential prescribing information: Surgifoam. Somerville, NJ, Johnson \& Johnson Wound Management. 2004.

[25] Baxter Healthcare Corporation. FloSeal Hemostatic Matrix: instructions for use. Fremont, CA, Baxter Healthcare Corporation. 2005.

[26] Nakayama T, Kumagai T. Gelatin allergy. Pediatrics 2004; 113(1 Pt 1); 170-1.

[27] Nakayama T, Aizawa C, Kuno-Sakai H. A clinical analysis of gelatin allergy and determination of its causal relationship to the previous administration of gelatin-containing acellular pertussis vaccine combined with diphtheria and tetanus toxoids. J Allergy Clin Immunol 1999; 103(2 Pt1); 321-5.

[28] Atrah HI. Fibrin glue. BMJ 1994; 308(6934): 933-4.

[29] Cederholm-Williams SA. Fibrin glue. BMJ 1994; 308(6943): 1570.

[30] Tredree R, Beierlein W, Debrix I, et al. Evaluating the differences between fibrin sealants: recommendations from an international advisory panel of hospital pharmacists. Eur J Hosp Pharm 2006; 1: 3-9.

[31] Scheule Am, Beierlein W, Wendel HP, et al. Aprotinin in fibrin tissue adhesives induces specific antibody response and increases antibody response of high-dose intravenous application. J Thorac Cardiovasc Surg 1999; 118: 348-58

[32] Scheule Am, Beierlein W, Wendel HP, et al. Fibrin sealant, aprotinin, and immune response in children undergoing operations for congenital heart disease. J Thorac Cardiovasc Surg 1998; 115: 883-9.

[33] Lynn AK, Yannas IV, Bonfield W. Antigenicity and immunogenicity of collagen. J Biomed Mater Res Part B: Appl Biomater 2004; 71(2): 343-54.

[34] British Medical Association, Royal Pharmaceutical Society of Great Britain. British National Formulary. London: BMA, RPS, 2007. (No 53.)

[35] Majerus PW, Broze GJ Jr, Miletrich JP, Tollefsen DM. Anticoagulant, thrombolytic, and antiplatelet drugs. In: Hardman JG, Limbird LE, Molinoff PB, Ruddon RW, Goodman Gilman, Eds. Goodman and Gilman's The pharmacological basis of therapeutics. New York, NY, McGraw-Hill. 1996: 1341-60.

[36] Levy JH. Management of anticoagulation and platelet receptor inhibitors in cardiac surgical patients. Available from: http: //www.heparininducedthrombocytopenia.com/revart3.asp
[37] Franchini M. Heparin-induced thrombocytopenia: an update. Thrombosis J 2005: 3: 14-8.

[38] Gruel Y, Pouplard C, Nguyen P, et al. Biological and clinical features of low-molecular-weight heparin-induced thrombocytopenia. $\mathrm{Br} \mathrm{J}$ Haematol 2003; 121(5): 786-92.

[39] Francis JL, Palmer GJ III, Moroose R, Drexler A. Comparison of bovine and porcine heparin in heparin antibody formation after cardiac surgery. Ann Thorac Surg 2003; 75(1): 17-22.

[40] Konkle BA, Bauer TL, Arepally G, et al. Heparin-induced thrombocytopenia: bovine versus porcine heparin in cardiopulmonary bypass surgery. Ann Thorac Surg 2001; 71(1): 1920-4.

[41] Rydberg EJ, Westfall JM, Nicholas RA. Low-molecular-weight heparin in preventing and treating DVT. Am Fam Physician 1999, 59(6): 1607-12.

[42] Greinacher A, Eichler P, Lietz T, Warkentin TE. Replacement of unfractionated heparin by low-molecular-weight heparin for postorthopedic surgery antithrombotic prophylaxis lowers the overall risk of symptomatic thrombosis because of a lower frequency of heparininduced thrombocytopenia. Blood 2005; 106(8): 2921-2.

[43] Walenga JM, Prechel M, Jeske WP, Bakhos M. Unfractionated heparin compared with low-molecular-weight heparin as related to heparin-induced thrombocytopenia. Curr Opin Pulm Med 2005; 11(5): 385-91.

[44] Anderson DG, Burdick JA, Langer R. Smart biomaterials. Science 2004; 305(5692): 1923-4.

[45] Langer R, Tirrell DA. Designing materials for biology and medicine. Nature 2004; 428(6982): 487-92.

[46] Ngan V, Oakley A. Collagen replacement therapy. Available at: dermnetnz.org/procedures/collagen.html

[47] Gillinoy AM. Heart valve surgery. Available from: www.clevel andclinic.org/heartcenter/pub/guide/disease/valve/valvetreatment.htm Anonymous. Prosthetic heart valves. Available at: cape.uwaterloo.ca/ che100projects/heart/files/testing.htm

[49] Allen GM. Surgical treatment of emphysema using bovine pericardium strips. AORN J 1006; 63(2): 373-88.

[50] Klepetko W. Surgical aspects and techniques of lung volume reduction surgery for severe emphysema. Eur Respir J 1999; 13(4): 91925 .

[51] Lemler MS, Ramaciotti C, Stromber D, Scott WA, Leorand SR. The extracardiac lateral tunnel Fontan, constructed with bovine pericardium: comparison with the extracardiac conduit Fontan. Am Heart J 2006; 151(4): 928-33.

[52] Lopes EJ, Santos TC, Jacobino M. Bovine pericardium in penile prosthesis reimplantation. International Brazil J Urol 2007; 33(1): 746.

[53] Gupta M, Lyon F, Singh AD, Rundle PA, Rennie IG. Bovine pericardium (Tutopatch) wrap for hydroxyapatite implants. Eye 2007 21(4): 476-9.

[54] Kapan S, Kapan M, Goksoy E, et al. Comparison of PTFE, pericardium bovine and fascia lata for repair of incisional hernia in rat model, experimental study. Hernia 2003; 7(1): 39-43.

[55] Dahm M, Husmann M, Eckhard-Mayer, Prufer D, Groh E, Oelert H. Relevance of immunologic reactions for tissue failure of bioprosthetic heart valves. Ann Thorac Surg 1995; 60(2suppl): S348-52.

[56] Konakci KZ, Bohle B, Blumer R, et al. Alpha-Gal on bioprostheses: xenograft immune response in cardiac surgery. Eur J Clin Invest 2005; 35(1): 17-23.

[57] Gong G, Seifter E, Lyman WD, Factor SM, Blau S, Frater RW. Bioprosthetic cardiac valve degeneration: role of inflammatory and immune reactions. J Heart Valve Dis 1993; 2(6): 684-93.

[58] Mire-Sluis AR, Barrett YC, Devanarayan V, et al. Recommendations for the design and optimization of immunoassays used in the detection of host antibodies against biotechnology products. J Immunol Methods 2004; 289(1-2): 1-16.

[59] European Medicines Agency (EMEA). Guideline on immunogenicity assessment of biotechnology-derived therapeutic proteins. London, European Medicines Agency (EMEA); 2007 